\title{
Erratum: Generalized particle dynamics: modifying the motion of particles and branes
}

\author{
Sudipta Das, ${ }^{a}$ Subir Ghosh, ${ }^{a}$ Jan-Willem van Holten ${ }^{b}$ and Supratik Pal ${ }^{a}$ \\ ${ }^{a}$ Physics and Applied Mathematics Unit, Indian Statistical Institute, \\ 203 B.T.Road, Kolkata 700108, India \\ ${ }^{b}$ Nikhef, \\ PO Box 41882, 1009 DB Amsterdam, Netherlands \\ E-mail: sudipta_jumaths@yahoo.co.in, sghosh@isical.ac.in, \\ t32@nikhef.nl, supratik_v@isical.ac.in
}

ERratum to: JHEP04(2009)115

Keywords: Classical Theories of Gravity, D-branes

The last paragraph of section 4 of is to be replaced by:

Negative $\boldsymbol{\beta}$. However, strikingly new behavior appears for negative $\beta$ with $l^{2}>|\beta| m^{2}$. This is seen in the Figure 1 for $\beta=-50$. The effective potential changes sign after some value of $r$, indicating a change in the behavior of the particle trajectory at some point. The cosmological implications of this sign-flip will be discussed in the next section.

This replacement has no consequences for the results of the remainder of the paper. 